Cahiers de recherches médiévales

Journal of medieval studies

$1 \mid 1996$

Croisades et idée de croisade à la fin du Moyen Âge

\title{
Un traité du droit des fiefs aux confins du Chartrain et de l'île de France (XIV ${ }^{\mathrm{e}}$ siècle)
}

Patricia Guyard

\section{(2) OpenEdition \\ Journals}

Édition électronique

URL : https://journals.openedition.org/crm/2537

DOI : $10.4000 / \mathrm{crm} .2537$

ISSN : 1955-2424

Éditeur

Honoré Champion

Édition imprimée

Date de publication : 15 janvier 1996

Pagination : 229-250

ISSN : 1272-9752

\section{Référence électronique}

Patricia Guyard, « Un traité du droit des fiefs aux confins du Chartrain et de l'île de France

(XIVe siècle) », Cahiers de recherches médiévales [En ligne], 1 | 1996, mis en ligne le 07 février 2008,

consulté le 15 décembre 2022. URL : http://journals.openedition.org/crm/2537 ; DOI : https://doi.org/

$10.4000 / \mathrm{crm} .2537$ 


\section{Un traité du droit des fiefs aux confins du chartrain et de l'Ile de France ( XIVe siècle)}

Le texte de droit coutumier et féodal qui fait l'objet de cette édition est extrait du cartulaire dit de Robert Mignon, une copie exécutée au XVe siècle d'un cartulaire élaboré vers 1350 et complété jusqu'en 1410 environ, conservée dans le fonds d'archives de la seigneurie du Tremblay-sur-Mauldre ${ }^{1}$. Ce cartulaire retrace un siècle d'acquisition, d'aliénation et de gestion de fiefs situés principalement dans le terroir du Tremblay et ses alentours immédiats, par trois membres de la famille Mignon, tous clercs du roi.

Jean Mignon ${ }^{2}$ se lança vers 1320 dans une politique d'acquisition foncière au Tremblay, dans la châtellenie de Maurepas. Son action fut très limitée territorialement mais fort efficace; à la fin de sa vie, en 1343 , il avait réuni près de cinquante parcelles et biens divers autours d'un petit patrimoine hérité de son père qui avait exploité à cens un manoir seigneurial et détenait quatre petits fiefs. Pour ce faire, Jean tira partie de sa connaissance du terroir et des nombreux détenteurs de ses fiefs ainsi que des avantages financiers que lui procuraient ses charges à la Chambre des comptes. Ainsi, il s'assura souvent la possession de biens de taille très variée en remboursement total ou partiel de prêt d'argent. Il fit également mettre en valeur fréquemment ses terres par ses neveux et nièces, tous alliés aux plus riches familles paysannes du village. Cela permit à son frère et à son neveu de reconstituer à leur profit les seigneuries du Tremblay et de La Hunière, démembrées les décennies précédentes entre plusieurs familles des environs. Robert Mignon ${ }^{3}$, frère de Jean, s'orienta vers l'achat de rentes en divers lieux à l'ouest de Paris, ne pouvant s'établir au Tremblay du vivant de son frère; il géra la succession de ce dernier de 1343 à 1355, date à laquelle il dut en aliéner une grande partie pour financer le collège fondé par Jean Mignon ${ }^{4}$. Néanmoins, il acquit la tour de La Hunière qui lui conférait la justice et le noyau de la seigneurie du lieu.

\footnotetext{
${ }^{1}$ Arch. dép. des Yvelines, $5 \mathrm{~J}$ 39. Le regeste du cartulaire ainsi qu'une étude de son contenu

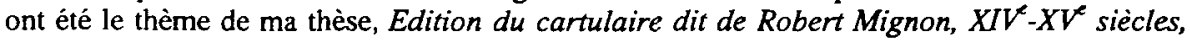
Paris, 1994 (thèse de l'Ecole des Chartes, dactyl.).

2 Jean Mignon mena de front une âpre carrière ecclésiastique troublée par les litiges liés à l'obtention de bénéfices (il devint archidiacre de Blois au diocèse de Chartres) et une carrière de clerc du roi à la Chambre des comptes; il fut désigné l'un des quatre maîtres clercs en 1320 (ordonnance de Vivier-en-Brie) et le resta jusqu'à sa mort en 1343.

${ }^{3}$ La carrière de Robert Mignon est en retrait par rapport à celle de son frère à la Chambre des comptes; auteur d'inventaires des comptes royaux, il resta simple clerc du roi, emploi qu'il perdit pour malversation financière.

${ }^{4}$ P. G., «La fondation du collège Mignon», Bibliothèque de l'Ecole des Chartes, 151, 1993, pp. 275-288.
}

Cahiers de Recherches Médiévales (XIII'-XV s.), 1, 1996 
Son fils, Michel ${ }^{5}$, recueillit en 1364 une succession certes difficile, mais restée assez compacte; son père s'était efforcé de vendre des fiefs excentrés dont la perte ne compromettait pas la possibilité de constituer à moyen terme les seigneuries du Tremblay et de La Hunière. Dans la désorganisation générale de la guerre et des troubles qui ravagèrent la région jusques en 1375 environ, Michel réussit à racheter les biens vendus par son père et à s'imposer comme seigneur du Tremblay et de la Hunière face à Pierre de Chevreuse, habile administrateur dans tous les rouages de l'Etat, et qui s'emparait en 1366 des biens d'Ingergier d'Amboise et de son gendre, prisonniers des Anglais depuis la bataille de Poitiers, dont dépendait le Tremblay. Ainsi constituées de débris éparpillés depuis un siècle et passablement ravagés durant ces années d'anarchie, les seigneuries trouvèrent une cohérence d'exploitation telle que malgré la reprise des hostilités contre les Anglais puis une succession partagée en 1456 entre les petits-neveux de Michel Mignon, de la famille des Culdöé, elles se perpétuèrent presque intactes, jusqu'à la Révolution.

La présence d'un texte de droit dans un corpus de copies d'actes ou de notices relatives à des transactions foncières ou aux formalités qui accompagnent la possession de fiefs n'a rien de très exceptionnelle tant la typologie des cartulaires et des sources qu'ils sont susceptibles de renfermer est diversifiée ${ }^{6}$; mais, dans le cas présent, plusieurs points peuvent attirer l'attention sur ce texte en dehors du fait qu'il soit inédit. L'un est son ampleur ; intitulé «Comment l'en se doibt demener en fiez», il s'étend sur deux feuillets et demi à raison de 36 lignes environ par page et comporte diverses annotations sur ses marges basses et latérales. A l'image de tous les autres textes du cartulaire, la compilation est sobre, nettement transcrite et découpée en paragraphes de longueurs très diverses: jusqu'au deuxième feuillet se poursuit un premier paragraphe traitant de la succession à un fief (condition du paiement du service et du rachat), suivi de deux autres alinéas concernant la recréance de fiefs lors de litiges avec un seigneur, puis d'une suite d'articles courts qui ne présentent aucun plan d'exposition.

Bien qu'il n'ait pas été possible de respecter dans l'édition le découpage un peu arbitraire du texte dans le cartulaire, on peut distinguer assez logiquement une cinquantaine d'articles. Ce caractère le différencie des simples notices écrites à l'occasion de quelque affaire et recopiées éventuellement à proximité de l'acte relatant la procédure, comme il s'en présente un exemple dans le cartulaire et à laquelle une note marginale dans le texte de droit renvoie afin de préciser la teneur d'un article sur la mise en foi de fiefs (article 12). Il s'agit bien ici d'une compilation sur un sujet précis dont elle aborde divers aspects, indépendante, a priori, des textes qui l'entourent sans pour autant être étrangère au corpus ${ }^{7}$.

\footnotetext{
5 Simple notaire du roi, Michel exerça notamment aux Requêtes du Palais ; mais, comme son oncle, il sut jouer des réseaux tissés au sein de la bourgeoisie parisienne, parmi les financiers des Comptes et les autres officiers de la Chancellerie.

6 Se reporter aux communications faites lors de la Table ronde organisée à ce sujet par l'Ecole des chartes et le CNRS, Les Cartulaires, Paris, Genève, 1993 (Mémoires et documents de l'Ecole des Chartes, t. 39).

${ }^{7}$ Ecrit sur le huitième cahier du cartulaire qui en compte dix, ce texte inaugure une série de 13 feuillets foliotés à la même cote en haut des recto, foliotation chaotique qui paraît suivre celle du cartulaire originel dans l'ensemble de la copie qui possède sa propre cotation conti-
} 
Face à un tel texte, on ne manque pas de penser à la relative rareté des écrits de droit féodal. Cette matière est abordée dans les coutumiers rédigés à partir du milieu du $\mathrm{XIII}^{\mathrm{e}}$ siècle et jusqu'au XVe siècle mais elle occupe bien peu de paragraphes, assez dispersés d'ailleurs, en regard du volume total des oeuvres et l'on s'y intéresse surtout au régime de succession ${ }^{8}$. Au XIV ${ }^{e}$ siècle apparaissent les premiers traités plus théoriques et spécialisés de praticiens tel Guillaume du Breuil qui écrit en 1330 le Stylus curie parlamenti consacrant un titre entier au droit des fiefs ${ }^{9}$. Aussi ce texte anonyme invite-t-il d'abord à une comparaison de forme, de structure et de contenu avec les compilations contemporaines de son élaboration afin de mettre en lumière quelques traits originaux. Mais son principal intérêt réside dans cette mise par écrit de règles de pure gestion foncière, de façon privée et délibérée, par des hommes qui, pour n'être pas juristes de formation, ont cependant acquis une connaissance juridique pour exercer leurs charges et gérer leurs biens ; texte assez précoce, atypique, ou plutôt illustration heureusement conservée, comme le cartulaire qui la contient, d'une pratique en réalité plus répandue qu'on ne le croit parmi les détenteurs de fiefs parce que nécessaire ? La situation géographique complexe du patrimoine de la famille Mignon, entre la Vicomté de Paris et le comté de Montfort, a sans doute favorisé la rédaction de ce texte mais d'autres facteurs peuvent être à l'origine de celuici.

Au-delà de la description du droit féodal dans cet écrit touchant incidemment quelques points d'actualité, comme la mutation monétaire, les mentions à diverses coutumes ou l'empreinte de l'enquête orale dont se nourrit visiblement l'auteur permettent d'apprécier la réalité des différences entre les usages coutumiers dès le XIV siècle et l'évolution du droit jusqu'à la rédaction des coutumes de Paris en 1510 et de Montfort en 1556.

Le texte émane de la même région que celle concernée par le cartulaire qui le renferme; il y est fait exclusivement mention de ressort de tribunaux et châtellenies où s'inscrivent toutes les mouvances des biens tenus par la famille Mignon à Montfort, Maurepas, Neauphle, Paris... Mais l'absence de mention d'auteur ou de date dans son corps comme dans le cartulaire rend très difficile la datation précise et la connaissance des conditions d'élaboration de ce texte. Néanmoins, il est plus que probable que l'un des trois clercs du roi ait été le commanditaire voire l'auteur de la

nue et complète au bas des feuillets ; ces feuillets sont consacrés à des textes très différents des autres actes du document : vente en 1355 par Robert Mignon de biens du Tremblay, tables des fiefs possédés par Robert avant et après la vente, rapport de l'enquête et de la franchise du paiement du droit d'amortissement par Michel Mignon, non noble, pour les fiefs acquis après 1323 au titre du privilège de bourgeoisie de Paris entre 1372 et 1374 avec copie de ce privilège et des quittances obtenues. L'étude de la constitution du cartulaire a amené à penser que ces documents étaient sans doute indépendants du cartulaire originel mais insérés dans celui-ci et recopiés, dans la foulée, lors de sa copie puisque leur matière intéresse directement le Tremblay et illustre les moments forts et les menaces pour le patrimoine de la famille Mignon.

8 Philippe de Beaumanoir, Coutumes de Beauvaisis, 1283, éd. A. Salmon, 1899-1900; Jean Boutillier, Somme rural, vers 1393, éd. Charondas Le Caron, 1603, pp. 477-480, 483-486, 488-494.

9 Tit. 28. Sequitur de materia homagiorum, éd. F. Aubert, 1909, p. 196-204. 
compilation puisque, bien qu'il s'agisse d'une copie certainement partielle, le cartulaire ne renferme dans un ordre particulièrement précis que des textes qui concernent ces trois personnes ${ }^{10}$. Elle pourrait être antérieure à 1330 comme le suggérerait une annotation à un article sur le délai maximal accordé à un détenteur de fief lésé dans sa propriété pour attaquer au possessoire. En effet, Guillaume du Breuil évoque dans le Stylus un délai de dix ans au Parlement d'après un arrêt de $1325^{11}$, délai qui a été porté en marge du texte étudié. En tout état de cause, il n'a pas paru incongru à l'auteur des notes marginales de renvoyer, afin de ne pas la répéter dans le traité, à une notice transcrite dans le cartulaire exposant les paroles dites lors de l'entrée en foi d'un seigneur en 1330 ; cela prouve au moins qu'il y a cohérence et validité de l'information à la date d'écriture de ces règles.

De compréhension difficile souvent, encore que les redites permettent d'élucider le sens d'articles obscurs, la compilation écrite dans le cartulaire de Robert Mignon n'est pas une traduction du latin au français mais bien une composition autonome dans ses sources comme dans sa langue. La forme de ce "glane» de droit n'a pu être rapproché d'aucun texte connu jusqu'ici. Certes, le sujet des actes qui environnent ce traité n'est guère différent de celui de quelques autres écrits de droit des fiefs copiés d'après ou sur des registres. Ainsi, «L'Usage des fiefs» du bailli de Vitry ${ }^{12}$ tire sa matière d'un registre des fiefs établi du 22 octobre 1354 au 21 janvier 1371. En éditant ce texte, François Olivier-Martin émit l'idée que son auteur fut un des clercs des fiefs présents auprès de baillis du roi envers qui les détenteurs de fiefs royaux accomplissaient certains de leurs devoirs ${ }^{13}$. Le livre Bertrand, qui provient de l'abbaye Saint-Martin-des-Champs, contient un texte de droit féodal en annexe d'une liste des fiefs et arrière-fiefs du prieuré ${ }^{14}$. Le texte édité ici est transcrit dans un cartulaire dont le sujet pour ainsi dire unique est la gestion des fiefs, et muni de deux tables récapitulatives de fiefs. La similitude d'environnement entre tous ces textes juridiques fait penser que le traité du cartulaire de Robert Mignon est un outil

10 Le cartulaire 5 J 39 comporte 336 textes et un censier; il en ressort, dans ce qu'il nous fait découvrir du cartulaire originel de Robert Mignon que ce demier a réuni les actes d'achat les aveux reçus et donnés et des récapitulations de fiefs de son défunt frère au Tremblay. Il a adjoint les aveux qu'il reçut et donna lui-même à l'occasion de la succession de son frère avant que son deuxième fils, Michel, ne les complète par ses propres actes, tous deux ajoutant les textes relatifs aux fiefs qu'ils acquéraient eux-mêmes hors du Tremblay. Ces trois parties ne se chevauchent pas, seul le huitième cahier où figure notre texte mélangeant des écrits de 1355 (sous Robert) et 1375 (sous Michel). Il est improbable que ces règles aient été écrites postérieurement à Michel Mignon et dans un environnement différent de celui que retrace cartulaire originel.

11 Guillaume du Breuil, Stylus curie Parlamenti, XVII, § 7 et XVIII, de causa novitatis, § 2 et 3 .

12 Usage des fiefs du bailliage de Vitry, éd. Fr. Olivier-Martin, «Textes inédits de droit champenois" dans Travaux juridiques et économiques de la faculté de droit de l'Université de Rennes, 1913.

13 G. Dupont-Ferrier, Gallia Regia ou état des officiers royaux des baillages et des sénéchaussées de 1328 a 1515. Paris, 1961, t. 6, pp. 175-205; de tels officiers sont mentionnés mais de façon discontinue et tardive.

14 G. Boulen et O. Martin, "Des fiez à l'usage de France, texte critique» dans Revue historique de droit, 1919, pp. 544-582, et 1920, pp. 135-158, 305-346. 
de travail en relation étroite avec les préoccupations immédiates qui président à l'écriture du registre même dont il porte les caractéristiques originales.

La principale source d'information paraît être orale ou issue d'une expérience réfléchie à part soi; de nombreux articles sont ponctués de l'expression «ce dient aucuns», des informateurs qui ne sont pas forcément des juristes, qui pourraient bien être des seigneurs locaux, des témoins de procédures. L'auteur procède souvent par comparaison ou juxtaposition d'usages de châtellenies locales sans s'attacher à une pratique coutumière précise ; cela renforce l'idée d'une utilisation directe pour régir des biens éparpillés dans tous les ressorts cités ${ }^{15}$. Loin d'être un style ${ }^{16}$, le traité s'attache à la seule gestion des fiefs, en dehors du tribunal, sur le terrain ; il n'aborde, parfois minutieusement, les cas de litiges que comme incidents éventuels dans la tenue d'un bien et pour préciser les rapports à entretenir entre seigneurs et vassaux, non la procédure judiciaire elle-même. Ces deux traits caractérisent le point de vue non d'un juriste mais bien d'un détenteur de fiefs, même s'il est juriste.

Nulle part enfin dans ce texte n'est fait mention d'une affaire ou d'une transaction précise qui aurait pu servir de support matériel à la confection des articles ou d'illustration à ceux-ci; cette indépendance envers toute référence à la pratique dans ou hors du cartulaire se double d'un style d'écriture qui énumère les règles sans les critiquer, ni commenter, ni illustrer d'exemples concrets. Les articles 18 et 38 sont seuls dotés d'exemples pour expliquer le mode de calcul du quint, requint et rachat, exemples, du reste, théoriques. Quelques répétitions laissent apparaitre des divergences (articles 6 et 38) et des notes marginales émettent de temps à autres des réserves (article 31) ou précisent des points ( article 6). Ce texte se contente donc d'exposer plus que d'expliciter la pratique; c'est un guide à l'usage du propriétaire foncier, comprenant même des paroles qui, formellement, accompagnent les démarches effectuées auprès du seigneur.

Voilà rendue sensible la différence de condition et d'esprit dans lesquelles ont été élaborés ce texte et le livre du bailli de Vitry pourtant à première vue fort proches l'un de l'autre par leur volume et leur matière. Dans le livre du bailli, où est plus forte l'empreinte du tribunal, la démarche est plus démonstrative pour démêler des points de droit délicats en ayant recours à des actes passés à Vitry que l'auteur n'hésite pas à citer et confronter ; le point de vue, l'expérience, les sources, la finalité du praticien dominent là où, dans l'autre texte, s'impose non le désir de débrouiller le droit et prévenir son évolution mais de l'utiliser dans un état présent, courant, pour

15 En celà il diffère du texte Des fiez à l'usage de France, qui lui est le plus proche par sa matière, montrant surtout pour Paris un état de la coutume très voisin de ce que ce texte en rapporte ; l'écart réside dans l'échelle géographique où ils s'inscrivent, France, Prévôté et Vicomté de Paris, Champagne pour l'un, c'est-à-dire grandes masses coutumières où l'on ne peut entrer bien loin dans le détail de procédure sans se heurter aux différences locales, et minuscules châtellenies à côté de Montfort et Paris pour l'autre, où, plus que de coutume, il s'agit d'usages locaux qui fixent le détail d'une amende, d'un service. Plus modeste dans son ressort, le texte de Robert Mignon n'en est pas moins plus scrupuleux du même coup et plus technique, livrant des détails qui n'apparaissent généralement pas à ce moment-là dans les rares théories de droit féodal.

${ }^{16} \mathrm{Par}$ contre, le traité adopte une démarche similaire aux styles de tribunaux qui décrivent pratiquement le déroulement des actions d'un procès; la matière du traité en est différente mais le plan adopté est bien celui de la description des étapes de la gestion des fiefs. 
une gestion simple et saine, y compris dans des cas complexes comme le rachat par le mari des fiefs de sa femme, discuté dans le texte de Vitry.

Le contenu entier du cartulaire dénote le respect scrupuleux des règles de tenue des fiefs et bien des aspects de la vie des trois clercs du roi pourraient corroborer cette impression et foumir quelques explications. Le premier tient aux fonctions qu'ils exercèrent; il ne semble pas qu'ils aient acquis quelque grade universitaire en droit ni détenu de charge judiciaire dans l'administration royale ; de la justice et des tribunaux, ils n'en ont que l'expérience, assez fournie du reste, de plaideurs ${ }^{17}$. Ils n'en sont pas moins habitués à l'écriture et au contrôle des actes qui, à la Chambre des comptes et à la Chancellerie, établissent obligations et droits fonciers et financiers $^{18}$. Pratique de l'écriture et environnement juridique incitent à considérer qu'écrire ou commander une compilation de droit ait pu être une évidence, une habitude professionnelle de clercs qui déborde sur le privé, qui pousse même à écrire un cartulaire quand tant d'autres seigneurs laïques se contentent d'un chartrier plus ou moins bien classé.

Quant à la possession foncière, la famille Mignon ne s'impose au Tremblay qu'à partir de Jean même si son père jouissait sans doute d'une honnête aisance. Avec Jean, la famille change de position sociale à tout point de vue; s'enrichissant modestement au service du roi et de l'Eglise, jouant le rôle de banquier auprès de seigneurs du lieu qu'elle connaît bien, saisissant toutes les opportunités d'acquisition sur les lieux mêmes de ses origines, il ne lui reste plus qu'à maîtriser parfaitement les formalités féodales, des rouages surtout financiers, pour satisfaire à ses récentes et lourdes obligations de détenteur de fiefs. A situation nouvelle, besoin exprès de s'enquérir de «son» droit ? Certes, mais on peut aller plus loin dans la portée d'un tel texte dont le fil conducteur est le rapport noué entre l'homme et le seigneur plus qu'entre le propriétaire et son bien, simple cause et enjeu des transactions.

On constate en effet que des points de droit évoqués avaient peu de chance d'intéresser l'un des membres de la famille Mignon (tels les articles portant sur la succession de nobles - que ne sont pas les trois clercs -, et de religieuses) ; c'est que toute détention de fiefs et d'arrière-fiefs induit un pouvoir sur des «hommes» ou de simples censitaires, implique la nécessaire capacité à se faire respecter mais aussi à arbitrer et trancher des conflits fonciers, intervenir dans le jeu des fiefs et contrôler l'évolution des mouvances en exerçant des prérogatives comme le retrait, toute opération qui se débat, et conclut éventuellement, à la cour du seigneur du fief que devinrent à leur tour les trois clercs. Des actes du cartulaire les montrent personnellement actifs sur ces questions, présidant réellement cette «cour», à la fois

${ }^{17}$ Les archives du Parlement de Paris livrent une activité soutenue en procédure civile, Jean pour défendre ses prétentions aux bénéfices ecclésiastiques et Robert et Michel, leur intérets dans la fondation et le financement du collège de Jean et quelques affaires familiales ; mais il y a quelques affaires foncières et féodales aussi, telle un retrait exercé sur un vassal par Michel Mignon en vertu de «la coutume des fiefs du lieu» (Arch. nat., X1c $35 n^{\circ}$ 94, 18 novembre 1373).

18 Jean et Robert ont dressé des inventaires à la Chambre des comptes, dont celui des comptes royaux édité par Ch.V. Langlois ; leur activité principale réside néanmoins dans le contrôle juridique et financier de toute pièce qui leur est présentée d'où il s'ensuit une familarité avec le droit. 
demeure et tribunal où hommes et tenanciers exposent leurs requêtes ou leurs défenses à l'un des clercs ou contre l'un de leurs hommes. La compétence des seigneurs est de plus en plus limitée, ce qui ressort aussi du texte où les cas de litiges ont tôt fait, sur simple demande du vassal désavouant le seigneur s'il y a saisie sans recréance du fief, de se porter au Parlement ou autre tribunal royal ; mais c'est la limite même des prétentions de ce texte: livrer les cas de figure généralement rencontrés par toute personne insérée à un niveau un peu élevé dans la hiérarchie des mouvances pour elle-même et comme responsable de sa mouvance et de tous ceux qui, à quelque titre, sont ses justiciables. Etre capable de se «demener» en fief est être conscient de cette double responsabilité.

On est loin cependant d'un dessein ambitieux; le droit des fiefs est celui de la propriété et la «trahison» du vassal qu'évoque le texte consiste le plus souvent au non-paiement d'un droit. Ce qui intéresse l'auteur est moins la nature du rapport homme-seigneur que les conséquences des transactions au sujet de ce qui les unit réellement et seulement : le bien. Succession, vente, échange, tout ce qui entraîne et réglemente l'accès à la propriété en sont le point fort et plus précisément, quand et pour quelle somme se monnaie cet accès. On oublierait presque que l'on achète l'acquiescement d'un seigneur qui use lui-même de ces procédés commandant la détention de la terre. L'exploitation et la transformation du «système féodal», commencé bien des décennies avant, sont donc particulièrement visibles mais aussi le respect du cadre formel du fief. $\mathrm{A}$ un formalisme de la fidélité et de l'hommage en succède un autre: la terre tend à ne plus être seulement l'expression d'un pouvoir en contrepartie de l'allégeance à un seigneur. Elle est de plus en plus considérée pour sa valeur intrinsèque, la rente qu'elle procure, l'ascension sociale qu'elle permet et symbolise pour son détenteur qui devient seigneur. Dans ce nouveau contexte, sa détention est toujours affaire de procédures entre deux personnes que canalise encore assez bien la hiérarchie des mouvances. Celle-ci consolide l'assise des nouveaux propriétaires au sein de la société détentrice des fiefs tout en garantissant et systématisant l'accès à une forme de propriété qui désormais obéit à une forme de «loi du marché».

Soucieux de leurs biens, trouvant dans ces règles des moyens d'action et de surveillance mais aussi des revenus "casuels», on peut penser que les trois clercs prirent particulièrement à coeur leur entreprise et leurs responsabilité pour en avoir consigné «le jeu». Nouvelle preuve de l'avidité et méticulosité à ne pas faire d'impair de la part des nouveaux propriétaires fonciers que sont généralement nombre d'officiers royaux, de bourgeois de Paris et d'autres grandes villes ? Possible, mais cette mise par écrit de droit féodal est originale dans ce milieu. Au moment où se multiplient les occasions de mutations, de jeux de fiefs et, partant, de procès, de tels écrits pourraient s'être avérés d'une aide précieuse pour gérer un patrimoine, apprécier une position dans la hiérarchie seigneuriale ou face à des communautés de tenanciers et autres banniers dès lors que les biens sont de quelque importance; mais force est de constater qu'on ne garde aucune trace de telle rédaction. En fait, le droit des fiefs lui-même tarde à être consigné, commence à l'être au milieu du XIVe siècle, quand le commerce de la terre augmente et s'ouvre, mais dans un milieu de praticiens que côtoient les membres de la famille Mignon. Ce qui a paru nécessaire à 
ces praticiens de noter à ce moment-là à leur usage a pu paraître tout aussi utile à consigner, connaître et avoir pour soi et près de soi par un simple propriétaire.

Du reste, une réelle complexité des ressorts locaux de châtellenies et coutumes contraint tout seigneur un peu doté en fiefs et arrière-fiefs à connaître les conséquences pratiques de ces enchevêtrements. De la châtellenie de Maurepas, elle-même dépendante de la seigneurie de Chevreuse, meut l'essentiel des terres et manoirs du Tremblay dont les marges sud et ouest cependant ressortissent au comté du Montfort tandis que Neauphle au nord étend ses mouvances dans les terroirs; assis dans le ressort de châtellenies autres que celle dont ils dépendent, selon quelle coutume tient-on les fiefs ? A trois reprises, le traité stipule qu'ils se servent et garantissent au prix fixé dans la châtellenie où ils sient et non dont ils meuvent ${ }^{19}$. A ces imbrications courantes de châtellenies s'ajoutent deux difficultés : l'une est que le Tremblay est situé sur la marge floue et mouvante qui sépare le comté de Montfort, à forte autonomie judiciaire, de la Prévôté et Vicomté de Paris au moment où est créé le ressort royal de Neauphle. La rivière de la Mauldre représente la limite entre Montfort et Paris laissant cependant à l'ouest Le Tremblay, séparé de Maurepas dont il dépend... La seconde est que, contrairement à ce qui se passe autours de Paris, le ressort diocésain de Paris ne coïncide pas pour le Pincerais avec celui de la Vicomté de Paris; le Tremblay, dans l'archidiaconné de Poissy, appartient au diocèse de Chartres ce qui laissera des traces dans la coutume car le droit a été l'affaire aussi des officialités dont le rôle s'est certes effacé mais dont l'influence a marqué le droit civil dans les coutumes, ce dont nous aurons à reparler.

Le texte offre une image assez fidèle des influences qui s'exercent localement et des parts respectives qu'elles régissent en matière de droit des fiefs. L'absence même de mention explicite à une coutume, à un ressort précis dans la plupart des articles en dit long sur les différences qu'on s'attendrait à voir là où pullulent des coutumes locales (Montfort, Neauphle, Chateron, Maurepas, Paris enfin) ; à part les allusions à «la coutume», au «dit-on», six articles seulement nomment des ressorts coutumiers dont un seul ecclésiastique, le diocèse de Chartres ${ }^{20}$. A priori, cela va dans le sens d'un droit assez uniforme, utilisant les mêmes moyens et principes généraux mélangeant les prestations personnelles (le service, l'entrée en foi), et foncières (le rachat, le quint-denier), délimitant la quotité des lots de successions; cette uniformité ne va pas tant de soi si on considère les étendues diverses que représente chacune des coutumes censées s'exercer localement; cette absence de mention à une coutume ne cacherait-elle pas parfois un compromis entre toutes ? Un compromis d'autant plus aisé que ce droit n'est pas codifié partout, à la même époque, et reste plus affaire de jurisprudence que de texte normatif ${ }^{21}$. Dans les principes généraux toutefois, c'est bien la communauté des règles qui l'emporte; l'auteur y est sans

19 Articles 3, 32 et 35 de l'édition. Un acte récapitulant le contenu du principal fief du Tremblay ( $n^{\circ} 125$ du regeste) délimite précisément dans l'énumération des tenanciers en censives ce qui ressort des châtellenies de "Chevreuse-Maurepas» et de Montfort, le tout ne mouvant que de Maurepas.

${ }_{20}$ Articles $n^{\circ} 1,2,14,45,51,53$.

21 C'est ainsi que quelques différences sont apparues en comparant des textes sur la coutume de fiefs de Paris au traité de Robert Mignon, telle l'absence du paiement du marc lors du rachat des fiefs. 
doute sensible ou y contribue-t-il plus ou moins volontairement en s'abstenant de citer un ressort précis qui pourrait faire croire à un usage particulier là où il est général.

A deux niveaux très différents cependant interviennent les mentions de coutumes pour différencier les usages. L'un indique une frontière réelle entre les grands ressorts juridictionnels que sont Montfort et Paris ou Chartres et qui perdurera jusqu'au delà de la rédaction des coutumes ou qui traduit une avancée de Paris bientôt adoptée par ses voisins; il est illustré par trois sujets.

Le don au conjoint sans contrepartie est autorisé au diocèse de Chartres, interdit à Paris où se confirme la méfiance envers de telles libéralités. L'influence de la juridiction gracieuse des officialités qui traitaient souvent de matière civile et testamentaire est telle que cette divergence de droit s'impose dans la pratique laïque chartraine et parisienne pour longtemps; la règle est aussi valable dans le texte de Robert Mignon dans la Vicomté de Paris et apparaît lors de la rédaction des coutumes de Paris et de Chartres ${ }^{22}$ telle qu'appliquée dans les diocèses respectifs. Or, l'archidiaconné chartraine de Poissy étant incluse dans la Vicomté de Paris, il y a localement flottement voire distorsion de la pratique, ce qui valut sans doute que ces ressorts soient précisés dans le traité. La rédaction des coutumes au $\mathrm{XVI}^{\mathrm{e}}$ sicle a clarifié le droit en privilégiant définitivement le ressort laïc.

La différence de dénomination et de montant du droit à acquitter pour la vente de fief entre Montfort et les autres coutumes n'est pas non plus sans fournir quelques enseignements. Plusieurs articles décrivent précisément le paiement du quint-denier car l'opération est rendue douteuse selon que le prix d'achat, réel ou non, est inscrit ou non à l'acte d'achat; mais on précise que, «partout où ailleurs il y a quint, à Montfort on paie le rachats. Le texte reflète l'état juridique intermédiaire entre l'apparition du droit spécifique qu'est le quint à Paris, vers 1250 , consacrant la plus grande facilité d'aliénation des fiefs, domaine où Paris semble avoir eu de l'avance sur les coutumes voisines, et sa généralisation au XIV ${ }^{\mathrm{e}}$ siècle en Normandie notamment tandis qu'il faudra attendre encore avant son implantation au Vexin et à Montfort où tout changement de vassal se concrétise par le rachat ; aussi n'est-on pas étonné de voir que l'auteur du texte se réfère précisément à la coutume parisienne en se donnant la peine de détailler la procédure et les montants à payer et l'on retrouvera exactement ces modalités là où le quint s'imposera.

Enfin, une note marginale au seul article portant sur le trouble dans la jouissance d'un bien, évoque la réglementation par le Parlement des actions possessoires

\footnotetext{
22 Bourdot de Richebourg, Nouveau coutumier général ou corps des coutumes générales et particulières de France, Paris, 1724, t. 3, Coutume de Chartres, chap. XVI «de donations et contrats faits entre vifs». Fr. Olivier Martin (Histoire de la coutume de la Prévôté et vicomté de Paris, Paris, 1926, t. 2, pp. 287-301) souligne que le droit parisien prohibe le don, sauf mutuel et égal de biens communs, à la différence du droit romain qui le tempère par sa révocabilité, au nom du maintien du patrimoine dans les familles d'où il provient et que cette interdiction s'étend après la rédaction des coutumes dans la Brie, à Senlis, à Chartres où la pratique était tolérée. De plus, l'auteur note que la nature ecclésiastique du ressort de cette

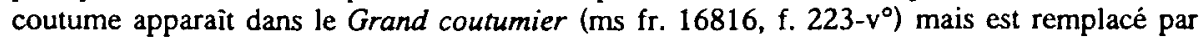
celui de la Prévôté de Paris dans les versions imprimées du texte. Le traité de Robert Mignon juxtapose les ressorts laïcs et ecclésiastiques (art. 45).
} 
et pétitoires qui s'affine non sans contradiction et tâtonnement au long du XIV siè- $^{\mathrm{e}}$ cle. Le texte ne distingue cependant pas nettement ces actions dans l'article 49 , se contentant de la partition entre la saisine et la propriété apparue à la fin du XII siècle ; sa teneur rappelle incidemment qu'au-delà l'an et jour, la saisine de fait revêt les protections de la possession sans aller plus loin dans la référence à la complainte en nouvelleté ou à la simple saisine qui peuvent s'exercer à la fin du XIV ${ }^{\mathrm{e}}$ siècle dans un délai de dix ans, ce que mentionne la note marginale ${ }^{23}$.

Dans ces trois cas, les distinctions coutumières d'origine différente - chartraine, parisienne, jurisprudentielle - ont tendance à s'estomper, dès le XIV ${ }^{\mathrm{e}}$ siècle pour la saisine et propriété, vers le $\mathrm{XV}^{\mathrm{e}}$ siècle pour le quint-denier et après la rédaction des coutumes pour le don. Parallèlement, on constate que les évolutions viennent de $\mathrm{Pa}$ ris ou bien y sont mises au point, et qu'elles s'imposent d'autant plus vite qu'elles traitent d'une matière nouvelle et technique. L'état du droit dans le traité est simple ; il ne s'attarde pas sur les rentes, par exemple, qui causent bien des soucis aux théoriciens et praticiens à Paris à la fin du siècle. Le cartulaire donne en fait de rares exemples de rentes véritables sinon le rachat, inhabituel pour l'époque, d'une rente constituée par Jean Mignon en 1323. La première inféodation de rente par un membre de la famille Mignon au Tremblay date seulement de 1400.

L'autre moment d'évoquer des coutumes précises ou orales, de les comparer aussi, est lorsqu'il s'agit de préciser le montant de diverses prestations. Le service «de cheval» dû par le vassal à la requête du seigneur, une seule fois dénommé ainsi dans le texte mais toujours de cette façon dans le cartulaire, n'est plus spécifié en nature comme il l'était encore dans les Constitucions $d u$ chastelet ou dans Des fiez $\grave{a}$ l'usage de France; la redevance est en argent et les montants divergent selon la châtellenie où est situé le fief. Cette taxe, selon A. Chédeville ${ }^{24}$, apparaît dans le pays chartrain vers 1218 , plus tôt en Anjou et Vendômois, au début et courant XI ${ }^{\mathbf{e}}$ siècle $^{25}$, tandis qu'elle se généralise à Paris et en Beauvaisis au XIII ${ }^{\mathrm{e}}$ siècle ; elle est encore rigoureusement levée au XIV siècle comme en témoignent nombre d'actes du cartulaire dans la région de Montfort. Fr. Olivier-Martin n'évoque pas le clivage entre localisation du fief et sa mouvance féodale et ressort judiciaire; il est sensible ici dans le fait que la valeur du service soit fixée par châtellenie, rare cas où la notion de châtellenie l'emporte sur celle de mouvance sous l'adage «les fiefs ne départent pas les châtellenies». On y peut voir les effets de démembrements de châtellenies dont des fiefs ont été transportés ou simplement ceux des imbrications d'inféodation. La conséquence au XIVe siècle est que le seigneur est tenu de respecter un montant fixé dans un ressort qui ne correspond presque jamais avec sa mouvance. D'autre part, les amendes dues en cas d'irrespect des règles ne varient pas, même dans une faible mesure, d'une châtellenie à l'autre. Dans les deux cas, les ressorts locaux des châtellenies à la fin du Moyen âge gardent quelques vestiges d'un pouvoir ancien du seigneur-châtelain, mais sans impact significatif sur les grands principes coutumiers et généralement sans exacte coïncidence avec les mouvances détenues par les seigneurs locaux.

23 Fr. Olivier Martin, op. cit, t. 2, pp. 56-63.

24 A. Chédeville, Chartres et ses campagnes, Paris, 1973.

$25 \mathrm{D}$. Barthélemy, La société dans le comté de Vendôme de l'an mil au XIVe siècle, Paris, 1993, pp. 850-853. 
Quelle sera l'évolution de ces pratiques ? Déjà très communes à cette petite région, elles gagnent peu à peu en précision et les sujets abordés ont plus d'ampleur ou de diversité. Aussi aurait-on tort d'attribuer à la seule rédaction des coutumes de $\mathrm{Pa}$ ris en 1510 la diffusion, quasiment mot pour mot, des règles de la capitale jusqu'à Montfort en 1556, puisque le mouvement est déjà visible au XIVe siècle. Pourtant, cette diffusion et sa consécration par la rédaction officielle des coutumes, n'allaient pas de soi, à lire les procès-verbaux dressés lors de la lecture des coutumes devant seigneurs et manants des villages des ressorts concernés; les vives oppositions qui s'élevèrent contre un article ou même l'appartenance à un ressort sont trop générales pour qu'on n'y prête pas un peu d'attention 26 .

Seules les coutumes de Montfort et Paris prévalent dans la région au $\mathrm{XVI}^{\mathrm{e}}$ siècle, ce qui ne nous étonne guère vu le niveau très modeste des citations des autres coutumes ou plutôt usages au XIV siècle. Pourtant, les villages se partagent mal entre les deux ressorts qui sont si proches.

La lecture des deux titres qui, dans les coutumes rédigées de Paris et Montfort, traitent du droit des fiefs, montre un souci sans doute plus grand de précision dans l'exposition des procédures et la description d'institutions peu répandues et surtout peu théorisées au XIV e siècle comme les rentes. Mais quelques évolutions sont notables, outre la diffusion du quint-denier dont il a été question plus haut; l'une concerne l'absence du paiement du marc d'argent qui accompagne le versement d'un an de revenu du fief comme mode de rachat possible de fief échu par succession collatérale. Au milieu du XIV ${ }^{\mathrm{e}}$ siècle les quittances de telles redevances sont nombreuses, mais on note qu'au baillage de Vitry, le marc n'est plus consigné parmi les trois «offres» du vassal pour payer le rachat ${ }^{27}$. La disparition s'est sans doute produite parce que,

26 Bourdot de Richebourg, op. cit. Les coutumes de Paris et Montfort sont très semblables; pourtant en 1556, Maurepas, Jouars, Neauphle-le-Vieux, Le Tremblay contestent, en vain, leur rattachement à la coutume de Montfort à la lecture de laquelle leurs représentants (manants, seigneurs, abbés) ont été appelés. Les localités absentes lors de cette lecture publique, bien que convoquées pour y adhérer, sont frontalières de la coutume de Paris rédigée dès 1510 en leur présence. Cette opposition ne s'appuie pas sur des motifs de droit coutumier qui aurairent été traités aussi dans le traité du XIV'e siècle; on ne peut donc, à partir de cette étude, en sonder la profondeur strictement juridique. Par contre, en 1580 , lors de la révision de la coutume de Paris, cette même lisière de contestation réapparait mais contre Paris. Le fonds du débat n'est pas d'ordre coutumier mais judiciaire ; le procureur du duc de Guise détenteur de la seigneurie de Chevreuse, expose à propos du Tremblay, plein fief de la châtellenie de Maurepas, terre "enclavée et assise en icelle» laquelle est unie au duché de Chevreuse, qu'il ne reconnait pas le prévôt de Paris en appel du bailli de Chevreuse mais le seul Parlement. Cette prétention découle-t-elle de l'érection de Chevreuse en duché survenue entre les deux rédactions ou est-elle plus ancienne? Le traité du XIV ${ }^{\mathrm{e}}$ siècle confirmerait plutôt l'ancienneté de la règle lorsqu'il décrit les appels du vassal contre le seigneur jusqu'au Parlement. En outre, Chevreuse, Maurepas et le Tremblay sont dans la liste des lieux réglés par la coutume de Paris en 1510 bien qu' Antoine de Canteleu, seigneur de Chevreuse, s'y opposait déjà ; cependant, dans le procès-verbal de la lecture de la coutume n'a pas été relevé le motif de droit que ce dernier allégua. Manifestement, il y a un désir d'autonomie moins coutumière que judiciaire de la part des terroirs dépendant de Chevreuse, comme de ceux dépendant de la châtellenie de Neauphle.

27 Usages des fiefs de Vitry..., art. 33. 
d'une valeur peu élevée en soi (le marc est couramment estimé à 60 s.p.), cette taxe due pour chaque fief est fort lourde lorsque le fief n'apporte pas lui-même un grand revenu et qu'une succession compte plusieurs dizaines de fiefs atomisés; il faut y voir un témoignage de l'extrême parcellisation des fiefs et de la réaction qui conduit à ne plus payer une taxe hors de proportion avec la valeur du fief, après un accommodement qu'évoque le texte de Robert Mignon. Dans son article 38 revenant sur le rachat traité précédemment pour décrire une pratique sans doute peu répandue, l'auteur du traité prend soin de l'attribuer à certains de ses informateurs. Cet accommodement introduit une forme de proportionnalité de la taxe selon la valeur du fief si celle-ci est inférieure à $10 \mathrm{l}$. ; ainsi, si le marc est estimé à $60 \mathrm{~s}$., ce qui en tient lieu ne doit pas excéder le tiers de revenu du fief. Même inflexion de la coutume de Paris et de Montfort pour le partage des fiefs entre deux héritiers mâles où le droit d'aînesse prévaut toujours mais où la part de l'aîné est ramenée des trois quarts aux deux tiers des biens ; elle est déjà présente dans le traité où l'auteur note les deux partitions possibles. Ainsi au XIV $\mathrm{V}^{\mathrm{e}}$ siècle se fixent les principales règles qui s'adaptent mieux à la parcellisation des fiefs avant qu'elles ne se retrouvent dans la rédaction officielle du XVIe siècle.

Enfin, c'est un décalage réel qui apparaît entre les deux périodes dans le cas de non-paiement de cens et champart; le texte stipule que, passé dix ans de non paiement, le seigneur peut s'attribuer le bien ${ }^{28}$ tandis qu'au $\mathrm{XVI}^{\mathrm{e}}$ siècle, le seigneur doit procéder par voie d'arrêt ou brandons sur «les fruits pendant par la racine» dès la troisième année sans que l'on sache bien s'il peut réclamer la totalité des arrérages ou la seule année en cours. Ainsi le seigneur du XIV ${ }^{\mathrm{e}}$ siècle peut ne rien percevoir pendant dix ans puis confisquer le bien. Au XVI considérée comme un manque de vigilance du seigneur; son droit au paiement du cens reste imprescriptible selon l'article 56 de la coutume de Montfort mais non le droit de percevoir les arrérages et il ne peut, semble-t-il, plus reprendre aussi aisément l'héritage.

Si les changements sont rares, ils n'en vont pas moins dans le même sens, celui d'un affaiblissement des droits du seigneur et des devoirs du feudataire ; relativement modeste certes mais très perceptible dans les textes normatifs entre les XIV $\mathrm{C}^{\mathrm{e}}$ et $\mathrm{XVI}^{\mathrm{e}}$ siècle, il contribue à l'assimilation de la tenure en fief à la propriété foncière pure et simple.

Document tourné vers la pratique féodale foncière et non vers la pratique judiciaire comme les autres textes connus à ce jour, cet écrit livre un étonnant témoignage des grandes étapes de la gestion d'un patrimoine où le système du fief, fortement «monétarisé» continue de présenter les avantages du contrôle de la détention foncière et l'inconvénient de la lourdeur et de la complexité. Il serait intéressant de confronter ce contenu normatif avec les actes de la pratique pour préciser, affiner ou infirmer l'adéquation d'une part entre le niveau théorique du texte et la gestion quotidienne du fief : aborde-t-il les thèmes courants que rencontre un détenteur de

28 Cela ne semble pas être une main-mise temporaire bien que l'article 47 précise que le seigneur n'acquiert pas la saisine contre son sujet et tenancier, car il s'agit plutôt ici d'une prescription du droit du censitaire à la détention de son bien dont il ne paie plus la redevance. 
Un traité du droit des fiefs...

fief; d'autre part entre ce qu'il préconise et la réalité du règlement des formalités et procédures.

Patricia Guyard 